\title{
Filling real hypersurfaces by pseudoholomorphic discs
}

\author{
Alexandre Sukhov* and Alexander Tumanov** \\ *Université des Sciences et Technologies de Lille, Laboratoire Paul Painlevé, U.F.R. de Mathématique, \\ 59655 Villeneuve d'Ascq, Cedex, France, sukhov@math.univ-lille1.fr \\ ** University of Illinois, Department of Mathematics 1409 West Green Street, Urbana, IL 61801, \\ USA, tumanov@math.uiuc.edu
}

Abstract. We study pseudoholomorphic discs with boundaries attached to a real hypersurface $E$ in an almost complex manifold. We give sufficient conditions for filling a one sided neighborhood of $E$ by the discs.

MSC: 32H02, 53C15.

Key words: almost complex manifold, Bishop disc.

\section{Introduction}

Since the fundamental work of Gromov [5] pseudoholomorphic curves have become an object of intensive research because of their remarkable applications in symplectic geometry and low dimensional topology; see [1,8] for numerous references. Pseudoholomorphic discs with boundaries in a prescribed real manifold play an important role. We call them the Bishop discs after Bishop [3], who introduced a method for constructing such discs. We refer the reader to the expository papers $[9,12,14]$ for the history and recent results on the Bishop discs in the case of the standard complex structure.

The main result of the paper is the following

Theorem 1.1 Let $E$ be a $C^{\infty}$-smooth real hypersurface in an almost complex manifold $(M, J)$. Assume that the set of all points where the Levi form of $E$ vanishes identically has empty interior. Also assume that E contains no J-complex hypersurface passing through a point $p \in E$. Then the Bishop discs of $E$ fill a one-sided neighborhood of $p$.

This paper is a continuation of our previous work [10], in which we prove a similar result for a manifold $M$ of complex dimension 2; see [10] for additional discussions. For the standard complex structure, a closely related result was obtained by Trépreau [11], who proved the forced one-sided extendibility of CR-functions rather than filling by the discs. The second 
author [13] proved an analogue for a generic submanifold $E$ of arbitrary codimension in $\mathbb{C}^{n}$. We must stress that $[11,13]$ don't need any assumptions on the Levi form.

In [10] we prove that if $M$ has complex dimension 2, and the hypersurface $E \subset M$ does not contain any $J$-complex curves, then the Bishop discs fill a one-sided neighborhood of each point of $E$. We don't need the Levi non-flatness assumption in [10], because in complex dimension 2 , if $E$ is Levi-flat, then it is foliated by $J$-complex hypersurfaces, that is, $J$-complex curves. This is no longer true in complex dimension 3 or higher due to an example by Ivashkovich and Rosay [6]. In this example a real hypersurface $E$ in an almost complex manifold of complex dimension 3 has identically vanishing Levi form but $E$ contains no complex hypersurfaces. Thus, in general, the vanishing of the Levi form of a real hypersurface does not imply integrability of the distribution of holomorphic tangent spaces. Nevertheless every Bishop disc of $E$ is contained in $E$, which is always true for real hypersurfaces with identically vanishing Levi form [7]. Hence the assumption on the Levi form can not be dropped in Theorem 1.1. It would be interesting to replace it by a weaker assumption.

Thus the non-integrability of an almost complex structure in complex dimension 3 or higher leads to a new phenomenon in the behavior of Bishop discs. This is the main motivation of our work.

Our method is based on our previous work [10]. For the convenience of the reader we use similar notations and terminology.

The paper was written for a special volume of JGA issued on the occasion of Gennadi Henkin's 65th birthday. The second author expresses deep gratitude to Henkin for advising him as a student, and for life-long support and inspiration. Both authors wish Henkin good health, happiness, and continuing success for many years to come.

\section{Preliminaries}

In this section we briefly recall some basic properties of almost complex manifolds. Throughout the paper, we assume by default that all relevant objects (manifolds, structures, etc. ) are smooth of class $C^{\infty}$.

\subsection{Almost complex structures}

Recall that a real $2 n$-dimensional manifold $M$ with a fixed $(1,1)$-tensor field $J$ satisfying $J^{2}=-I d$ is called an almost complex manifold; the tensor field $J$ is called an almost complex structure.

A real submanifold $Y$ of an almost complex manifold $(M, J)$ is called $J$-complex if for every point $p \in Y$, the tangent space $T_{p} Y$ is a $J$-complex subspace of $T_{p} M$, that is, $J(p) T_{p} Y=$ $T_{p} Y$. A $J$-complex hypersurface is a $J$-complex submanifold of real dimension $2 n-2$.

We denote by $\mathbb{D}$ the unit disc in $\mathbb{C}$ and by $J_{s t}$ the standard complex structure in $\mathbb{C}^{n}$. Let $(M, J)$ be an almost complex manifold and let $f$ be a smooth map from $\mathbb{D}$ into $M$. As usual 
we say that $f$ is $J$-holomorphic if $d f \circ J_{s t}=J \circ d f$. We call such a map $f$ a $J$-holomorphic disc or a pseudoholomorphic disc.

An almost complex manifold $(M, J)$ of complex dimension $n$ can be locally viewed as the unit ball $\mathbb{B}$ in $\mathbb{C}^{n}$ equipped with a small almost complex deformation of $J_{s t}$. More precisely, given point $p \in M$, for every $\delta_{0}>0$, and every $k \geq 0$ there is a neighborhood $U$ of $p$ and a smooth coordinate chart $z: U \longrightarrow \mathbb{B}$ such that $z(p)=0, d z(p) \circ J(p) \circ d z^{-1}(0)=J_{s t}$, and the direct image $z_{*}(J):=d z \circ J \circ d z^{-1}$ satisfies the inequality $\left\|z_{*}(J)-J_{s t}\right\|_{C^{k}(\overline{\mathbb{B}})} \leq \delta_{0}$.

Definition 2.1 Consider a $C^{2}$ function $u$ on $M$. Let $p$ be a point of $M$ and $v \in T_{p} M$ be $a$ tangent vector. The Levi form of the function $u$ at $p$ evaluated on $v$ is defined by the equality $L^{J}(u)(p)(v):=-d\left(J^{*} d u\right)(v, J v)(p)$. The Levi form of a real hypersurface $E=\{\rho=0\} \subset M$ at $p \in E$ is the conformal class of the Levi form $L^{J}(\rho)(p)$ of the defining function $\rho$ on the $J$-holomorphic tangent space $H_{p}^{J} E:=T_{p} E \cap J T_{p} E$.

It is well known (see, e. g., [6]) that $L^{J}(u)(p)(v)=\Delta(u \circ f)(0)$ where $f$ is an arbitrary $J$-holomorphic disc in $M$ such that $f(0)=p$ and $d f(0)\left(\partial_{\operatorname{Re} \zeta}\right)=v$ (here $\zeta$ is the standard complex coordinate variable in $\mathbb{C}$ ). We also point out that the Levi form is invariant with respect to $J$-biholomorphisms: if $\Phi$ is a $\left(J, J^{\prime}\right)$-holomorphic diffeomorphism from $(M, J)$ into $\left(M^{\prime}, J^{\prime}\right)$, then $L^{J}(u)(p)(v)=L^{J^{\prime}}\left(u \circ \Phi^{-1}\right)(\Phi(p))(d \Phi(p)(v))$.

We use only the quadratic Levi form defined above. Similarly, one could define a bilinear version of the Levi form. Then vanishing of the bilinear Levi form of a real hypersurface $E$ is equivalent to Frobenius-integrability of the $J$-holomorphic tangent bundle $H^{J} E$. However, in the almost complex case, the bilinear Levi form is not necessarily symmetric, so vanishing of the quadratic Levi form does not imply that $E$ is foliated by $J$-complex hypersurfaces.

We refer the reader to $[1,4,8,9]$ for further details on almost complex structures, CRmanifolds, and pseudoholomorphic discs.

\subsection{Normal form of an almost complex structure along a pseudo- holomorphic disc}

Let $J$ be a smooth almost complex structure on a small enough neighborhood $U$ of the origin in $\mathbb{C}^{n}$ and $J(0)=J_{s t}$. Denote by $z=\left(z_{1}, \ldots, z_{n}\right)$ the standard coordinates in $\mathbb{C}^{n}$. In the matrix computations throughout this paper we always view $z$ as a column. We will use the following notation: $\bar{\partial}:=\frac{\partial}{\partial \bar{\zeta}}, \partial:=\frac{\partial}{\partial \zeta}$.

Then a map $z: \mathbb{D} \longrightarrow U$ is $J$-holomorphic if and only if it satisfies the following system of partial differential equations (the Cauchy - Riemann equations):

$$
\bar{\partial} z-A(z) \overline{\partial z}=0
$$

where $A(z)$ is the complex $n \times n$ matrix defined by

$$
A(z) v=\left(J_{s t}+J(z)\right)^{-1}\left(J_{s t}-J(z)\right)(\bar{v}) .
$$


It is easy to see that the right-hand side is $\mathbb{C}$-linear in $v \in \mathbb{C}^{n}$ with respect to the standard structure $J_{s t}$, hence $A(z)$ is well defined. Since $J(0)=J_{s t}$, we have $A(0)=0$. Furthermore, by shrinking the neighborhood of the origin where the initial disc $z$ is contained, we can assume that given positive $k$ the $C^{k}$ norm of $A$ is as small as we need. This simple argument is used throughout the paper. We denote by $e_{n}$ the last vector of the standard basis of $\mathbb{C}^{n}$ that is

$$
e_{n}=(0, \ldots, 1)
$$

Lemma 2.2 Let $z_{0}$ be a J-holomorphic disc sufficiently close in the $C^{k}$ norm for some $k \geq 2$ to the disc $\zeta \mapsto \zeta e_{n}, \zeta \in \mathbb{D}$. Then there exists a local coordinate diffeomorphism in a neighborhood of $z_{0}(\overline{\mathbb{D}})$ such that in the new coordinates we have $z_{0}(\zeta)=\zeta e_{n}, \zeta \in \mathbb{D}$. Furthermore, in the new coordinates we have $A\left(\zeta e_{n}\right)=0, A_{z}\left(\zeta e_{n}\right)=0$ for $\zeta \in \mathbb{D}$.

This statement is proved in [10] in the case of complex dimension 2. The proof goes through in any dimension with obvious modifications, so we omit it.

Let $\rho$ be a real function in a neighborhood of the origin in the space $\mathbb{C}^{n}$ equipped with a smooth almost complex structure $J$. Even if $J(0)=J_{s t}$, the Levi for of $\rho$ with respect to $J$ at the origin does not necessarily coincide with the Levi form with respect to $J_{s t}$. However, if the coordinates are normalized according the previous lemma, the Levi forms of $\rho$ with respect to $J$ and $J_{s t}$ are the same (see, e. g., [10]).

Lemma 2.3 Assume that $A(0)=A_{z}(0)=0$. Then the Levi form of $\rho$ at the origin with respect to the structure $J$ coincides with the Levi form of $\rho$ at the origin with respect to the structure $J_{s t}$.

\section{Bishop discs and the Bishop equation}

Let $(M, J)$ be a smooth almost complex manifold of complex dimension $n$ and $E$ a real generating submanifold of codimension $d$ in $M$. Recall that a $J$-holomorphic disc $f: \mathbb{D} \longrightarrow$ $M$ continuous on $\overline{\mathbb{D}}$ is called a Bishop disc if $f(b \mathbb{D}) \subset E$, where $b \mathbb{D}$ denotes the boundary of $\mathbb{D}$.

If $p \in E$ and a Bishop disc $f$ additionally satisfies the condition $f(1)=p$ then we say that $f$ is attached to $E$ at $p$.

Let $\rho=\left(\rho_{1}, \ldots, \rho_{d}\right)$ be a local defining function of $E$ in a neighborhood $U$ of a point $p$ where local coordinates $z$ are fixed. Then a smooth map $z: \mathbb{D} \longrightarrow U$ continuous on $\overline{\mathbb{D}}$ is a Bishop disc if and only if it satisfies the system (1) of partial differential equations with the boundary condition $(\rho \circ z)(\zeta)=0, \zeta \in b \mathbb{D}$. We call this boundary value problem the Bishop equation by analogy with the case of $\mathbb{C}^{n}$. The neighborhood $U$ is supposed to be small enough so we call such Bishop discs small. The Bishop equation can be easily solved by the Implicit Function Theorem using functional properties of the standard integral operators. This implies the existence and local parametrization of Bishop discs attached to E. The following existence result is obtained in [10]. 
Proposition 3.1 Given positive non-integralk the set $\mathcal{A}_{p}^{J}(E)$ of J-holomorphic Bishop discs of $E$ of class $C^{k}(\overline{\mathbb{D}})$ satisfying $z(1)=p$ is a Banach manifold and its tangent space at the constant disc $z \equiv p$ is canonically isomorphic to the space $\left(C^{k}(\overline{\mathbb{D}}) \cap \mathcal{O}_{1}(\mathbb{D})\right)^{n-d}$ of vector functions valued in $\mathbb{C}^{n-d}$, holomorphic (with respect to $J_{\text {st }}$ ) on $\mathbb{D}$, of class $C^{k}$ on $\overline{\mathbb{D}}$ and vanishing at the point 1 . The manifold $\mathcal{A}_{p}^{J}(E)$ depends smoothly on a point $p$ and a deformation of the complex structure $J$.

The following example is due to Ivashkovich and Rosay ([6], section 6).

Example 1. Consider in $\mathbb{C}^{3}$ with the standard complex coordinates $z=\left(z_{1}, z_{2}, z_{3}\right)$ the almost complex structure $J$ such that the equations (1) for a $J$-holomorphic disc $z: \zeta \mapsto z(\zeta)$ have the form

$$
\begin{aligned}
& \bar{\partial} z_{j}=0, j=1,2 \\
& \bar{\partial} z_{3}=\bar{z}_{1} \overline{\partial z_{2}}
\end{aligned}
$$

An equivalent definition of $J$ as a real matrix function is given in [6]. It is shown in [6] that the real hypersurface $E=\left\{\operatorname{Im} z_{3}=0\right\}$ contains no $J$-complex hypersurfaces. On the other hand it is easy to see that the Levi form of $E$ with respect to $J$ vanishes identically at every point and according to the general result [7] all Bishop discs of $E$ are contained in $E$. In fact, in this example the Bishop discs $z$ attached to $E$ at the origin can be described as follows.

Since $z$ is $J$-holomorphic, then $z_{1}$ and $z_{2}$ are holomorphic functions on the unit disc in the usual sense. Then the equation for $z_{3}$ implies $z_{3}=\phi+\bar{\psi}$, where $\phi$ is holomorphic and $\psi(\zeta)=\int_{1}^{\zeta} z_{1}(\tau) z_{2}^{\prime}(\tau) d \tau$.

Since $z$ is a Bishop disc for $E$ then $\left.\operatorname{Im} z_{3}\right|_{b \mathbb{D}}=0$. Since $z_{3}$ is harmonic, then $\operatorname{Im} z_{3}$ vanishes on $\mathbb{D}$ identically, that is, $z(\mathbb{D}) \subset E$. Then $z_{3}(\zeta)=2 \operatorname{Re} \int_{1}^{\zeta} z_{1}(\tau) z_{2}^{\prime}(\tau) d \tau$, and $z_{1}$ and $z_{2}$ are arbitrary holomorphic functions in $\mathbb{D}$ with $z_{1}(1)=z_{2}(1)=0$.

This description implies that the evaluation map

$$
\mathcal{F}: \mathcal{A}_{0}^{J}(E) \ni z \mapsto z(-1)=\left(z_{1}(-1), z_{2}(-1), 2 \operatorname{Re} \int_{1}^{-1} z_{1}(\tau) z_{2}^{\prime}(\tau) d \tau\right) \in E
$$

has the rank 5 except at the constant disc $z=0$. It also implies that the range of $\mathcal{F}$ is all of $E$. Thus, all Bishop discs attached to $E$ at the origin lie in $E$ and fill all of it.

Example 1 shows that the notion of the defect of a Bishop disc introduced in [13] does not make sense in the almost complex setting. According to [13], for a small Bishop disc $z$ through a fixed point $p=z(1)$ of a real generic manifold $E \subset\left(\mathbf{C}^{n}, J_{s t}\right)$, the spaces of infinitesimal perturbations of $z^{\prime}(1)$ and those of $q=z(-1)$ have the same codimension in the respective ambient spaces. This codimension is called the defect of the disc $z$. In Example 1 , for a non-constant disc $z$, we found that the map $z \mapsto z(-1)$ has the rank 5 whereas the map $z \mapsto z^{\prime}(1)$ has the rank at most 4 because $z(\mathbb{D}) \subset E$. Hence the approach based on the defect fails in this example.

Baouendi, Rothschild, and Trépreau [2] interpret the defect of a disc in terms of its lifts to the conormal bundle of $E$ in the cotangent bundle $T^{*} \mathbb{C}^{n}$. Although an almost complex 
structure admits natural lifts to the cotangent bundle of an almost complex manifold (see, e.g., [16]), obviously, they don't give rise to the correct notions of the defect of Bishop discs.

The following simple consequence of Proposition 3.1 reduces the proof of the main result to constructing a single transverse Bishop disc similarly to [10, 13].

Proposition 3.2 Let $E$ be a real hypersurface in an almost complex manifold $(M, J)$. Suppose that there exists $z \in \mathcal{A}_{p}^{J}(E)$ such that the normal derivative vector $d z\left(\left.\partial_{\operatorname{Re}} \zeta\right|_{1}\right)$ is not tangent to $E$ at $p$ (that is the Bishop disc $z$ is attached to $E$ at $p$ transversally). Then Bishop discs of E fill a one-sided neighborhood of $p \in E$.

In some special cases the condition of transversality can be easily verified. For instance, if $E$ is a pseudoconvex hypersurface or satisfies standard finite type conditions [10]. However, this is a substantially more subtile task to prove the existence of a transversal Bishop disc without these additional assumptions. This is our main goal in the present paper.

Our key tool is the following statement.

Theorem 3.3 Let $E$ be a generating submanifold in an almost complex manifold $(M, J)$ and let $f_{0}$ be a small enough embedded Bishop disc attached to $E$ at a point $p \in E$ and tangent to $E$ at $p$. Suppose that every Bishop disc $f$ attached at $p$ and close enough to $f_{0}$ also is tangent to $E$ at $p$. Then the Levi form of every defining function of $E$ vanishes identically on the holomorphic tangent space of $E$ at every point of the boundary of the disc $f_{0}$.

In the present paper we will use this proposition only in the case where $E$ is a hypersurface.

Corollary 3.4 Let $E$ be a real hypersurface in an almost complex manifold $(M, J)$ and let $f_{0}$ be a small enough embedded Bishop disc attached to $E$ at a point $p \in E$ and tangent to $E$ at $p$. Suppose that every Bishop disc $f$ attached at $p$ and close enough to $f_{0}$ also is tangent to $E$ at $p$. Then the Levi form of $E$ vanishes identically along the boundary of the disc $f_{0}$.

In the hypothesis of Theorem 3.3 we will suppose that

$$
E=\left\{\rho=\left(\rho_{1}, \ldots, \rho_{d}\right)=0\right\}
$$

is a real generating $C^{\infty}$-smooth submanifold of codimension $d$ in an almost complex manifold $(M, J)$ of complex dimension n. Since all our considerations are purely local, we work in local coordinates. For technical reasons it is more convenient to consider Bishop discs attached to $E$ at the point $e_{n}$ so we suppose that $E$ contains it. Our almost complex structure $J$ is viewed as a real $(2 n \times 2 n)$-matrix function $J: z \mapsto J(z)$. We also can assume that $J\left(e_{n}\right)=J_{s t}$. We view a defining function $\rho$ of $E$ as a vector function valued in the space $\mathbb{R}^{d}$ of real columns. Recall also that a map $\zeta \mapsto z(\zeta)$ from the unit disc $\mathbb{D}$ to $\mathbb{C}^{n}$ is $J$-holomorphic 
if and only if it satisfies (1). We stress that we consider here only maps valued in a small enough neighborhood of the point $e_{n}$.

Consider small embedded $J$-holomorphic Bishop discs attached to $E$ at the point $e_{n}$. For every such a disc $z_{0}$ there exists a local diffeomorphism such that in the new coordinates we have $z_{0}(\zeta)=\zeta e_{n}, \zeta \in \mathbb{D}$ (see Lemma 2.2). We denote again by $J$ the representation of our almost complex structure in the new coordinates, $J\left(e_{n}\right)=J_{s t}$.

We establish the following coordinate version of Theorem 3.3.

Theorem 3.5 Suppose that for the Bishop disc $z_{0}: \zeta \mapsto \zeta e_{n}, \zeta \in \mathbb{D}$, we have $A \circ z_{0}=0$ and $A_{z} \circ z_{0}=0$ (here $A$ is the matrix from the Cauchy-Riemann equations (1)) and

$$
\begin{aligned}
& \rho_{z_{j}}^{k}\left(e_{n}\right)=\delta_{j}^{k}, k, j=1, \ldots, d, \\
& \rho_{z_{j}}^{k}\left(e_{n}\right)=0, k=1, \ldots, d, j=d+1, . ., n
\end{aligned}
$$

where $\delta_{j}^{k}$ denotes Kroneker's symbol. Assume that the derivatives $A_{\bar{z}}$ and the second derivatives of the functions $\rho^{k}$ are small on the disc $z_{0}(\overline{\mathbb{D}})$. Suppose further that for every Bishop disc $z: \zeta \mapsto z(\zeta)$ with $z(1)=z_{0}(1)=e_{n}$ close enough to $z_{0}$ we have

$$
\partial z_{k}(1)=0, k=1, \ldots, d
$$

Then $L^{J}\left(\rho^{k}\right)(p)(v)=0, k=1, \ldots, d$ for every point $p \in z_{0}(b \mathbb{D})$ and every vector $v \in H_{p}^{J}(E)$.

Remark 1. The assumptions of smallness of the derivatives of $A$ and $\rho$ are automatically satisfied because the original disc is small and the change of coordinates stretches it to the unit disc. The normalization condition also can be achieved by a suitable change of coordinates which does not affect previous assumptions (Lemma 2.2). So Theorem 3.3 is a consequence of Theorem 3.5.

Remark 2. We point out that in the above coordinates the Levi form of $\rho$ with respect to $J_{s t}$ coincides with the Levi form with respect to $J$ at any point of the set $z_{0}(b \mathrm{D})$ (Lemma $2.3)$.

We provide the proof of Theorem 3.5 in the next two sections.

\section{Infinitesimal analysis of the Bishop equation}

In this section we linearize Bishop's equation and then solve the obtained linear boundary problem for an elliptic system of partial differential equations. This gives a convenient parametrization of the tangent space $T_{z_{0}} \mathcal{A}_{p}^{J}(E), p=e_{n}$. In particular this allows to reformulate the moment condition of tangency of all Bishop discs attached at $p$ as a condition of vanishing of a non-linear map $\Phi$ defined on the Banach manifold $\mathcal{A}_{p}^{J}(E)$. All the statements of this section are proved in [10] for the case of complex dimension 2 and the proofs remain valid in the general case with obvious changes. For the convenience of readers we outline here the main steps of this construction. 


\subsection{Linearized Bishop equation}

Consider a Bishop disc $z: \zeta \mapsto z(\zeta), \zeta \in \mathbb{D}$, attached to $E$ at $e_{n}$ and close enough to $z_{0}$. Then this disc satisfies the following boundary problem:

$$
\begin{aligned}
& \bar{\partial} z=A \overline{\partial z}, \\
& \left.\rho \circ z\right|_{b \mathbb{D}}=0 .
\end{aligned}
$$

Recall that we only deal with small Bishop discs, so we can assume that for every fixed non-integral $k>0$ the norm $\|A\|_{C^{k}}$ is small enough.

Now we introduce solutions $\dot{z}$ of the corresponding linearized problem:

$$
\begin{aligned}
& \bar{\partial} \dot{z}=\dot{A}(z) \overline{\partial z}+A \overline{\partial \dot{z}}, \\
& \left.\operatorname{Re}\left(\rho_{z}(z) \dot{z}\right)\right|_{b \mathbb{D}}=0,
\end{aligned}
$$

where $\rho_{z}$ is the Jacobi matrix:

$$
\rho_{z}=\left(\begin{array}{ccc}
\rho_{z_{1}}^{1} & \ldots & \rho_{z_{n}}^{1} \\
\ldots & \ldots & \ldots \\
\rho_{z_{1}}^{d} & \ldots & \rho_{z_{n}}^{d}
\end{array}\right)
$$

The symbol $\dot{A}$ is defined as follows. Consider the map $z \mapsto A \circ z$ defined on the space of smooth discs $z: \mathbb{D} \longrightarrow \mathbb{C}^{n}$. Then $\dot{A}(z)$ denotes the value of the Frechet derivative of this map at $z$ and $\dot{z}$ (a tangent vector at $z$ ): $\dot{A}=A_{z} \dot{z}+A_{\bar{z}} \overline{\dot{z}}$. As usual we call the solutions $\dot{z}$ of this linearized system the infinitesimal variations of the disc $z$.

In order to obtain a convenient description of the space of solutions of the linearized Bishop equation, following [10], we make suitable changes of dependent variables. Namely, put $I^{\prime}=(I-A \bar{A})^{-1}$ and $\lambda=\rho_{z} I^{\prime}+\rho_{\bar{z}} \overline{I^{\prime} A}$,

$$
\lambda=\left(\begin{array}{ccc}
\lambda_{1}^{1} & \ldots & \lambda_{n}^{1} \\
\ldots & \ldots & \ldots \\
\lambda_{1}^{d} & \ldots & \lambda_{n}^{d}
\end{array}\right)
$$

Consider also the matrix $\Lambda$

$$
\Lambda=\left(\begin{array}{ccccc}
\lambda_{1}^{1} & \ldots & \lambda_{d+1}^{1} & \ldots & \lambda_{n}^{1} \\
\ldots & \ldots & \ldots & \ldots & \ldots \\
\lambda_{1}^{d} & \ldots & \lambda_{d+1}^{d} & \ldots & \lambda_{n}^{d} \\
0 & \ldots & 1 & \ldots & 0 \\
\ldots & \ldots & \ldots & \ldots & \ldots \\
0 & \ldots & 0 & \ldots & 1
\end{array}\right)
$$

We assume that $\Lambda$ is smoothly extended on the disc $\mathbb{D}$. Introduce the change of variable

$$
v=\Lambda(\dot{z}-A \bar{z}), \quad v=\left(v_{1}, \ldots, v_{n}\right) .
$$


Proposition 4.1 The new dependent variable $v$ has the following properties.

(i) $v$ is a solution of the equation

$$
\bar{\partial} v=B_{1} v+B_{2} \bar{v}
$$

where $B_{j}=B_{j}(z)$ are certain smooth $(n \times n)$ - matrices.

(ii) $v$ satisfies the boundary condition

$$
\operatorname{Re} v=\left(\begin{array}{l}
0 \\
u
\end{array}\right), v(1)=0
$$

where $u: b \mathbb{D} \longrightarrow \mathbb{R}^{n-d}, u=\left(u_{d+1}, \ldots, u_{n}\right)$, is an arbitrary smooth vector function with $u(1)=0$.

(iii) We have

$$
\partial v_{k}(1)=0
$$

for $k=1, \ldots, d$.

(iv) $\dot{B}_{1}-(\bar{\partial} \dot{\Lambda}) \Lambda^{-1}$ is a linear combination of $\dot{z}, \overline{\dot{z}}$ with smooth coefficients depending on $\zeta$, which we write in the form

$$
\dot{B}_{1}=(\bar{\partial} \dot{\Lambda}) \Lambda^{-1} \bmod (\dot{z}, \bar{z})
$$

and similarly $\dot{B}_{2}$ is a linear combination of $\dot{z}, \overline{\dot{z}}$ and $\overline{\partial \dot{z}}$ with smooth coefficients depending on $\zeta$ :

$$
\dot{B}_{2}=0 \bmod (\dot{z}, \overline{\dot{z}}, \bar{\partial} \overline{\dot{z}})
$$

The proof is given in [10].

\subsection{Parametrization of the solution space of the linearized Bishop equation}

In order to describe solutions of the linearized Bishop equation we need some integral operators which we briefly recall here. The Cauchy-Green transform is defined by

$$
T f(\zeta)=\frac{1}{2 \pi i} \iint_{\mathbb{D}} \frac{f(\tau)}{\tau-\zeta} d \tau \wedge d \bar{\tau} .
$$

It is classical ([15], p. 56, Theorem 1.32) that for every integral $m \geq 0$ and $0<\alpha<1$ the linear map $T: C^{m+\alpha}(\overline{\mathbb{D}}) \longrightarrow C^{m+\alpha+1}(\overline{\mathbb{D}})$ is bounded.

We use the following notation for the Cauchy integral

$$
K f(\zeta)=\frac{1}{2 \pi i} \int_{b \mathbb{D}} \frac{f(\tau) d \tau}{\tau-\zeta}
$$


We also set

$$
K_{1} u:=K u-(K u)(1)
$$

The boundary problem stated in Proposition 4.1 (i), (ii) was solved in [10]. Its solution has the form

$$
v=v_{0}+R_{1} v_{0}+R_{2} \bar{v}_{0},
$$

Here the resolvent operators $R_{j}$ are $\mathbb{C}$-linear bounded operators $C^{m+\alpha} \longrightarrow C^{m+\alpha+1}$ for any integral $m \geq 0$ and $0<\alpha<1$ and

$$
v_{0}=\left(\begin{array}{c}
0 \\
\varphi
\end{array}\right)
$$

where $\varphi$ is a holomorphic vector function given by

$$
\varphi=2 K_{1} u .
$$

One can view $R_{j}$ as $(n \times n)$-matrices with operator entries. Furthermore, in [10] an expansion of $R_{1}$ to a sum of operator series is obtained and studied. Every term of this series is a composition of several integral operators, which are suitable modification of the Cauchy-Green operator $T$, and the operators of left multiplication by the matrices $B_{j}, \bar{B}_{j}$.

Now we can interpret the condition

$$
\partial v_{1}(1)=\ldots=\partial v_{d}(1)=0
$$

(see (iii) in Proposition 4.1) similarly to [10]. We have

$$
v_{j}=\sum_{k=d+1}^{n} R_{1}^{j k} \varphi_{k}+\sum_{k=d+1}^{n} R_{2}^{j k} \bar{\varphi}_{k}
$$

for $j=1, \ldots, d$. Here $R_{1}^{j k}$ and $R_{2}^{j k}$ denote the $(\mathrm{jk})$ matrix entries of the matrix operators $R_{1}$ and $R_{2}$ respectively. Thus the condition (8) means that

$$
\sum_{k=d+1}^{n} \partial R_{1}^{j k} \varphi_{k}(1)+\sum_{k=d+1}^{n} \partial R_{2}^{j k} \bar{\varphi}_{k}(1)=0
$$

Since the first term is $\mathbb{C}$-linear and the second is anti-linear, we get $\sum_{k=d+1}^{n} \partial R_{1}^{j k} \varphi_{k}(1)=0$ for all $\varphi$ with $\varphi(1)=0$. Hence,

$$
\partial R_{1}^{j k} \varphi_{k}(1)=0, j=1, \ldots, d, k=d+1, \ldots, n
$$

for all holomorphic functions $\varphi_{k}$ with $\varphi_{k}(1)=0$.

Now we rewrite the condition (9) in a more convenient form. The method of [10] is based on the smoothing properties of the operators involved to the expansion of $R_{1}$. Differentiation 
of these operators in (9) leads to certain singular integral operators whose kernels have singularities at the point 1 . These operators loose the smoothing property at this point. In [10] we overcome this technical difficulty by introducing suitable function spaces. The idea is to consider spaces of functions which are smooth on $\mathbb{D}$ except the point 1 . Then we also need to adapt the notion of a bounded linear operator to this class of spaces.

Given integral $m \geq 0$ and $0<\alpha<1$ the spaces $C_{1}^{m+\alpha}(\overline{\mathbb{D}})$ and $C_{1}^{m+\alpha}(b \mathrm{D})$ are defined as spaces of functions which are of class $C^{m+\alpha}$ on $\overline{\mathbb{D}} \backslash\{1\}$. More precisely $f \in C_{1}^{m+\alpha}(\overline{\mathbb{D}})$ if $f \in L^{\infty}(\mathbb{D})$ and for every $\varepsilon>0$ we have $\left.f\right|_{\overline{\mathbb{D}} \backslash \mathbb{B}(1, \varepsilon)} \in C^{m+\alpha}(\overline{\mathbb{D}} \backslash \mathbb{B}(1, \varepsilon))$, where $\mathbb{B}(1, \varepsilon)$ denotes the disc of radius $\varepsilon$ centered at 1 . The space $C_{1}^{m+\alpha}(b \mathrm{DD})$ is defined similarly. As in [10] we say that $P$ is a bounded linear operator $C_{1}^{m+\alpha}(\overline{\mathrm{D}}) \longrightarrow C_{1}^{k+\beta}(\overline{\mathrm{D}})$ for integral $m, k \geq 0$ and $0<\alpha, \beta<1$, if for every $\varepsilon>0$ there exists a constant $C=C(\varepsilon)>0$ such that for every $f \in C_{1}^{m+\alpha}$ we have

$$
\|P f\|_{C^{k+\beta}(\mathbb{D} \backslash \mathbb{B}(1,2 \varepsilon))}+\|P f\|_{L^{\infty}(\mathbb{D})} \leq C\left(\|f\|_{C^{m+\alpha}(\mathbb{D} \backslash \mathbb{B}(1, \varepsilon))}+\|f\|_{L^{\infty}(\mathbb{D})}\right) .
$$

Similarly are defined bounded operators $C^{m+\alpha}(\overline{\mathrm{D}}) \longrightarrow C_{1}^{k+\beta}(\overline{\mathrm{D}})$ and when we have $b \mathbb{D}$ in place of $\mathbb{D}$.

Definition 4.2 We write $P_{1} \sim P_{2}$ for two operators $P_{1}$ and $P_{2}$ if $P_{1}-P_{2}$ is a bounded operator $C^{1+\alpha}(\overline{\mathbb{D}}) \cap \mathcal{O}(\mathbb{D}) \longrightarrow C_{1}^{2+\alpha}(b \mathbb{D})$. Here $\mathcal{O}(\mathbb{D})$ denotes the space of holomorphic (in the usual sense) functions on $\mathbb{D}$.

The proof of the following three propositions is given in [10]. They form crucial steps of our reduction.

First, we reduce the condition (9) to a vanishing of certain non-linear map on the space of Bishop discs.

Proposition 4.3 The condition (9) is equivalent to the fact that for any $j=1, \ldots, d$ and $k=d+1, \ldots, n$ we have

$$
\Phi^{j k}(z):=\left.T\left(B_{1}+\Omega\right)^{j k}\right|_{b \mathbb{D}}=0
$$

where a map $\Omega: z \mapsto \Omega(z)$ associates to $z$ an $(n \times n)$-matrix with operator entries and the index $(j k)$ refers to the matrix entry. This property holds for every Bishop disc z close enough to $z_{0}$ with $z(1)=e_{n}$.

A precise description and analysis of the map $\Omega$ is contained in [10]. Here we just point out that in the notation of [10] the this map admits an expansion $\Omega=\bar{B}_{2} \mu(\tau)+\Sigma^{\prime}+\Sigma^{\prime \prime}$, where $\Sigma^{\prime}$ and $\Sigma^{\prime \prime}$ are the sums of certain operators series. Every term of these series is a composition of certain singular integral operators and operators of the left multiplication by the matrices $B_{j}, \bar{B}_{j}$. These series converge in the space $C^{\mathbf{1}-\beta}(\overline{\mathbb{D}})$ for every $0<\beta<1$.

The map $\Phi=\left(\Phi^{k}\right)$ is defined in a neighborhood of the disc $z_{0}$ on a Banach manifold $\mathcal{A}_{p}^{J}(E)$ of $J$-holomorphic Bishop discs attached to $E$ at the point $p=e_{n}$. It follows from (10) that the map $\Phi$ vanishes identically. Hence its Fréchet derivative $\dot{\Phi}$ at $z_{0}$ also does. This leads to the second step (the linearization): 
Proposition 4.4 For the Fréchet derivative of $\Phi$ we have

$$
\dot{\Phi}=\left(\dot{\Phi}^{j k}\right)=\left(\left.T\left[\dot{B}_{1}+\dot{\Omega}\right]^{j k}\right|_{b \mathbb{D}}\right)=0 .
$$

where as above $j=1, \ldots, d, k=d+1, \ldots, n$.

In other words $\dot{\Phi}(\dot{z})=0$, for every $\dot{z} \in T_{z_{0}} \mathcal{A}_{p}^{J}(E)$.

In view of the parametrization of the tangent space $T_{z_{0}} \mathcal{A}_{p}^{J}(E)$ given by (7) we can view $\dot{\Phi}$ as an $\mathbb{R}$-linear operator applied to a vector-function $\varphi \in\left(\mathcal{O}(\mathbb{D}) \cap C^{1+\alpha}(\overline{\mathbb{D}})\right)^{n-d}, 0<\alpha<1$.

Proposition 4.5 The condition (11) implies that

$$
\left.T\left(I_{0} \dot{B}_{1}\right)^{j k}\right|_{b \mathbb{D}} \sim 0
$$

$j=1, \ldots, d, k=d+1, \ldots, n$. Here $I_{0}=I+a_{1}+a_{2} \mu, \mu(\tau)=\left(\frac{\tau-1}{\bar{\tau}-1}\right)^{2}$, and each matrix function $a_{j}$ is of class $C_{1}^{m+\alpha}(\overline{\mathbb{D}})$ for all integral $m \geq 0,0<\alpha<1$.

The matrices $a_{j}$ here are written in [10] in the terms of the expansion of $\Omega$ mentioned after Proposition 4.3. The matrices $a_{j}$ are small, so $I_{0}$ is close to $I$. In particular the matrix $I_{0}$ is invertible. The equivalence $\sim$ in (12) is understood in the sense of Definition 4.2.

\section{Infinitesimal analysis of the operator $\Phi$}

Now we are able to prove Theorem 3.5. The idea is to remove the terms contained in $\Omega$ in (11). The equivalence $\sim$ of operators is always understood in the sense of Definition 4.2.

Consider $[T, b]:=T \circ b-b \circ T$ the commutator of the operator $T$ and the operator of multiplication by a matrix $b$. The next technical statement also is due to [10].

Lemma 5.1 Let $b \in C_{1}^{m+\alpha}(\overline{\mathbb{D}})$ for any all integral $m \geq 0,0<\alpha<1$. Then $[T, b]$ defines a bounded operator $C^{s}(\overline{\mathbb{D}}) \longrightarrow C_{1}^{s+2}(\overline{\mathbb{D}})$ for any non-integral $s>0$.

In other words $T \circ b \sim b \circ T$, which we use below. The first step is the following

Proposition 5.2 We have

$$
\left.\left(T \dot{B}_{1}\right)^{j k}\right|_{b \mathbb{D}} \sim 0
$$

for $j=1, \ldots, d, k=d+1, \ldots, n$. 
Proof : Using the commutator argument and Lemma 5.1 we obtain from (12) that

$$
\left.\left(I_{0} T \dot{B}_{1}\right)^{j k}\right|_{b \mathbb{D}} \sim 0
$$

for $j=1, \ldots, d$ and $k=d+1, \ldots, n$. Then

$$
\left.0 \sim\left(I_{0} T \dot{B}_{1}\right)^{j k}\right|_{b \mathbb{D}}=\sum_{s=1}^{n} I_{0}^{j s} T \dot{B}_{1}^{s k} .
$$

Since

$$
\dot{\Lambda}=\left(\begin{array}{ccccc}
\dot{\lambda}_{1}^{1} & \ldots & \dot{\lambda}_{d}^{1} & \ldots & \dot{\lambda}_{n}^{1} \\
\ldots & \ldots & \ldots & \ldots & \ldots \\
\dot{\lambda}_{1}^{d} & \ldots & \dot{\lambda}_{d}^{d} & \ldots & \dot{\lambda}_{n}^{d} \\
0 & \ldots & 0 & \ldots & 0 \\
\ldots & \ldots & \ldots & \ldots & \ldots \\
0 & \ldots & 0 & \ldots & 0
\end{array}\right)
$$

and by (3) we have

$$
\dot{B}_{1}=(\bar{\partial} \dot{\Lambda}) \Lambda^{-1} \bmod (\dot{z}, \bar{z}),
$$

we obtain that

$$
\left.T \dot{B}_{1}^{s k}\right|_{b \mathbb{D}} \sim 0
$$

for $s=d+1, \ldots, n-1$ and every $k$. Therefore

$$
\left.\sum_{s=1}^{d} I_{0}^{j s}\left(T \dot{B}_{1}\right)^{s k}\right|_{b \mathbb{D}} \sim 0
$$

for $j=1, \ldots, d, k=d+1, \ldots, n$. Recall that the matrix $I_{0}$ is close to $I$ (see the end of Section 4 after Proposition 4.5), so the matrix $\left(I_{0}^{j s}\right)_{j, s=1, \ldots, d}$ is invertible. This implies (13).

On the disc $z_{0}$ we have

$$
\begin{gathered}
\lambda \circ z_{0}=\left(\begin{array}{ccc}
\rho_{z_{1}}^{1} & \ldots & \rho_{z_{n}}^{1} \\
\ldots & \ldots & \ldots \\
\rho_{z_{1}}^{d} & \ldots & \rho_{z_{n}}^{d}
\end{array}\right) \\
\Lambda \circ z_{0}=\left(\begin{array}{ccccc}
\rho_{z_{1}}^{1} & \ldots & \rho_{d+1}^{1} & \ldots & \rho_{z_{n}}^{1} \\
\ldots & \ldots & \ldots & \ldots & \ldots \\
\rho_{z_{1}}^{d} & \ldots & \rho_{d+1}^{d} & \ldots & \rho_{z_{n}}^{d} \\
0 & \ldots & 1 & \ldots & 0 \\
\ldots & \ldots & \ldots & \ldots & \ldots \\
0 & \ldots & 0 & \ldots & 1
\end{array}\right)
\end{gathered}
$$

Using the obvious block structure of the above matrix, we have

$$
\Lambda \circ z_{0}=\left(\begin{array}{cc}
X & Y \\
0 & I
\end{array}\right) \quad\left(\Lambda \circ z_{0}\right)^{-1}=\left(\begin{array}{cc}
X^{-1} & -X^{-1} Y \\
0 & I
\end{array}\right),
$$


where $X=\left(\rho_{z_{k}}^{j}\right)_{j, k=1, \ldots, d}$. Now using the condition (13) and Lemma 5.1, we conclude that

$$
\left.T(\bar{\partial} \dot{\lambda})\right|_{b \mathbb{D}}\left(\begin{array}{c}
-X^{-1} Y \\
I
\end{array}\right) \sim 0
$$

Finally we need to express $\dot{\lambda}$ in terms of $\varphi$. We use the notation $\lambda^{k}$ for the row $\lambda^{k}=$ $\left(\lambda_{1}^{k}, \ldots, \lambda_{n}^{k}\right)$ of the matrix $\lambda$. The condition $A_{z} \circ z_{0}=0$ implies

$$
\dot{\lambda}^{k}=\left(\rho_{z}^{k}\right)^{\cdot}+\rho_{\bar{z}}^{k} \dot{\bar{A}}=\left(a^{k} \dot{z}+b^{k} \overline{\dot{z}}\right)^{t}
$$

where $a^{k}=\rho_{z z}^{k}+\rho \frac{k}{\bar{z}} \bar{A}_{z}$ and $b^{k}=\rho_{z \bar{z}}^{k}$ and the upper index $t$ denotes the matrix transposition.

We have $\dot{z}=\Lambda^{-1} v_{0}+(S)$ where $(S)$ denotes smoother terms. Then

$$
\dot{z} \sim \Lambda^{-1}\left(\begin{array}{l}
0 \\
\varphi
\end{array}\right)=\left(\begin{array}{cc}
X^{-1} & -X^{-1} Y \\
0 & I
\end{array}\right)\left(\begin{array}{l}
0 \\
\varphi
\end{array}\right)
$$

By the Cauchy-Green formula, $T \bar{\partial} \varphi=0$ and $T \bar{\partial} \bar{\varphi}=\bar{\varphi}$. Again by Lemma 5.1 we obtain

$$
\left.T \bar{\partial} \dot{\lambda}^{k}\right|_{b \mathbb{D}} \sim\left(a^{k} T \bar{\partial} \dot{z}+b^{k} T \bar{\partial} \dot{\dot{z}}\right)^{t} \sim\left(b^{k} \overline{\dot{z}}\right)^{t} \sim\left(\overline{\dot{z}}_{1}, \ldots, \overline{\dot{z}}_{n}\right)\left(\begin{array}{ccc}
\rho_{z_{1} \bar{z}_{1}}^{k} & \ldots & \rho_{z_{n} \bar{z}_{1}}^{k} \\
\ldots & \ldots & \ldots \\
\rho_{z_{1} \bar{z}_{n}}^{k} & \ldots & \rho_{z_{n} \bar{z}_{n}}^{k}
\end{array}\right)
$$

Then the conditions (14) can be rewritten in the form

$$
\bar{\varphi}^{t} L\left(\rho^{k}\right) \sim 0, k=1, \ldots, d
$$

where

$$
L\left(\rho^{k}\right)=\left(\begin{array}{ll}
-\left(\bar{X}^{-1} \bar{Y}\right)^{t} & I
\end{array}\right)\left(\begin{array}{ccc}
\rho_{z_{1} \bar{z}_{1}}^{k} & \cdots & \rho_{z_{n} \bar{z}_{1}}^{k} \\
\cdots & \cdots & \cdots \\
\rho_{z_{1} \bar{z}_{n}}^{k} & \cdots & \rho_{z_{n} \bar{z}_{n}}^{k}
\end{array}\right)\left(\begin{array}{c}
-X^{-1} Y \\
I
\end{array}\right)
$$

Thus the product $\bar{\varphi}^{t} L\left(\rho^{k}\right)$ is of class $C_{1}^{2+\alpha}(b \mathbb{D})$ for any vector function $\varphi \in\left(C^{1+\alpha}(\overline{\mathbb{D}}) \cap\right.$ $\mathcal{O}(\mathbb{D}))^{n-d}$. Hence the matrix $L\left(\rho^{k}\right)$ vanishes identically on the boundary of the disc $z_{0}$. But this is exactly the matrix of the restriction of the Levi form of the function $\rho^{k}$ on the holomorphic tangent space (with respect to $J_{s t}$ ) at a point of $z_{0}(b \mathrm{DD})$.

Recall that the conditions $A \circ z_{0}=0$ and $A_{z} \circ z_{0}=0$ imply that the Levi form of $\rho^{k}$ with respect to the structure $J$ coincides with the Levi form of $\rho^{k}$ with respect to the structure $J_{s t}$ at every point of the boundary of the disc $z_{0}$ (Lemma 2.3). Thus the Levi form with respect to the structure $J$ of every defining function of $E$ vanishes on the boundary of the disc $z_{0}$, as desired.

This proves Theorem 3.5. 


\section{The case of degenerate rank}

We return to the case where $E=\{\rho=0\}$ is a hypersurface. In this section we study the situation where the boundaries of the Bishop discs attached to $E$ through a fixed point do not cover an open set in $E$. The method of [10] can be easily adapted to this case.

Proposition 6.1 Suppose that the boundaries of J-holomorphic discs $\zeta \mapsto z(\zeta)$ with $z(1)=$ $e_{n}$ attached to $E$ and close to the disc $z_{0}: \zeta \mapsto \zeta e_{n}, \zeta \in \mathbb{D}$, do not cover an open set in $E$. Then for every $\zeta_{0} \in b \mathrm{D}, \zeta_{0} \neq 1$ there exists a J-complex hypersurface near the point $\zeta_{0} e_{n}$ completely contained in E.

Proof : $\quad$ Fix a point $\zeta_{0} \neq 1$ in $b \mathbb{D}$. For every Bishop disc $z(\zeta)$ close enough to $z_{0}(\zeta)$ we define the evaluation map $\mathcal{F}_{\zeta_{0}}: z \mapsto z\left(\zeta_{0}\right)$. Its Fréchet derivative $\dot{\mathcal{F}}_{\zeta_{0}}$ at $z_{0}$ is given by $\dot{\mathcal{F}}_{\zeta_{0}}: \dot{z} \mapsto \dot{z}\left(\zeta_{0}\right)$ where $\dot{z}$ is an infinitesimal perturbation of $z_{0}$.

By assumption the boundaries of discs do not cover an open subset of $E$. Therefore $\operatorname{rank} \dot{\mathcal{F}}_{\zeta_{0}} \leq 2 n-2$ for all $\zeta_{0}$. First we show that rank $\dot{\mathcal{F}}_{\zeta_{0}} \geq 2 n-2$.

Lemma 6.2 For every vector $\left(q_{2}, \ldots, q_{n}\right) \in \mathbb{C}^{n-1}$ there exists $\dot{z}=\left(\dot{z}_{1}, \dot{z}_{2}, \ldots, \dot{z}_{n}\right)$ with $\left(\dot{z}_{2}\left(\zeta_{0}\right), \ldots, \dot{z}_{n}\left(\zeta_{0}\right)\right)=\left(q_{2}, \ldots, q_{n}\right)$.

Proof : $\quad$ Recall again that $A=0$ and $A_{z}=0$ on the disc $z_{0}$ and

$$
\begin{aligned}
& \dot{z}=\Lambda^{-1} v, \\
& v=\left(\begin{array}{c}
0 \\
\varphi
\end{array}\right)+R_{1}\left(\begin{array}{c}
0 \\
\varphi
\end{array}\right)+R_{2}\left(\begin{array}{c}
0 \\
\bar{\varphi}
\end{array}\right),
\end{aligned}
$$

where $\varphi=\left(\varphi_{2}, \ldots, \varphi_{n}\right)$ is an arbitrary holomorphic vector function valued in $\mathbb{C}^{n-1}$ satisfying $\varphi(1)=0$. Then for $j=2, \ldots, n$

$$
\dot{z}_{j}=\varphi_{j}+\sum_{k=2}^{n} R_{1}^{j k} \varphi_{k}+\sum_{k=2}^{n} R_{2}^{j k} \bar{\varphi}_{k}
$$

Plugging $\zeta=\zeta_{0}$, we get for $j=2, \ldots, n$

$$
\dot{z}_{j}\left(\zeta_{0}\right)=\varphi_{j}\left(\zeta_{0}\right)+\sum_{k=2}^{n} \iint_{\mathbb{D}} a_{j k}^{1}(\zeta) \varphi_{k}(\zeta) d \zeta \wedge d \bar{\zeta}+\sum_{k=2}^{n} \iint_{\mathbb{D}} a_{j k}^{2}(\zeta) \overline{\varphi_{k}(\zeta)} d \zeta \wedge d \bar{\zeta}
$$

where $a_{j k}^{i}$ are integrable functions in $\mathbb{D}$ (this integral representation follows from the analysis of the expansion of $R_{j}$ into operator series in [10]). If the rank of the map $\varphi \mapsto$ $\left(\dot{z}_{2}\left(\zeta_{0}\right), \ldots, \dot{z}_{n}\left(\zeta_{0}\right)\right)$ is smaller than or equal to $2 n-3$, then there exists a vector $c=$ $\left(c_{2}, \ldots, c_{n}\right) \in \mathbb{C}^{n-1} \backslash\{0\}$ such that for every $\varphi$ we have $\operatorname{Re}\left(\sum_{j=2}^{n} c_{j} \dot{z}_{j}\left(\zeta_{0}\right)\right)=0$. Then for some $b_{j}^{1}, b_{j}^{2} \in L^{1}(\mathbb{D})$ we have

$$
2 \operatorname{Re}\left(\sum_{j=2}^{n} c_{j} \varphi_{j}\left(\zeta_{0}\right)\right)+\sum_{j=2}^{n} \iint_{\mathbb{D}} b_{j}^{1}(\zeta) \varphi_{j}(\zeta) d \zeta \wedge d \bar{\zeta}+\sum_{j=2}^{n} \iint_{\mathbb{D}} b_{j}^{2}(\zeta) \overline{\varphi_{j}(\zeta)} d \zeta \wedge d \bar{\zeta}=0
$$


Splitting into linear and anti-linear parts we get

$$
\sum_{j}\left(c_{j} \varphi_{j}\left(\zeta_{0}\right)+\iint_{\mathbb{D}} b_{j}^{1}(\zeta) \varphi_{j}(\zeta) d \zeta \wedge d \bar{\zeta}\right)=0 .
$$

Similarly to $[10]$ this implies that $c_{j}=0$ for every $j=2, \ldots, n$. Indeed, take for instance $\varphi_{j}=0, j=3, \ldots, n$ and $\varphi_{2}=\psi^{n}$, where $\psi$ has a peak at $\zeta_{0}$, that is, $\psi(0)=0, \psi\left(\zeta_{0}\right)=1$ and $|\psi(\zeta)|<1$ for $\zeta \in \overline{\mathbb{D}} \backslash\left\{\zeta_{0}\right\}$. Then passing to the limit as $n \longrightarrow \infty$ we obtain that $c_{2}=0$. We proceed similarly for other $c_{j}$. The obtained contradiction proves the lemma.

Thus the rank of the map $\varphi \mapsto \dot{z}\left(\zeta_{0}\right)$ is equal to $2 n-2$. Hence there exist vectors $d^{1}, d^{2} \in \mathbb{C}^{n-1}$ such that

$$
\dot{z}_{1}\left(\zeta_{0}\right)=\sum_{j=2}^{n} d_{j}^{1} \dot{z}_{j}\left(\zeta_{0}\right)+\sum_{j=2}^{n} d_{j}^{2} \overline{\dot{z}_{j}\left(\zeta_{0}\right)}
$$

for all $\varphi$. The equality $\dot{z}=\Lambda^{-1} v$ implies

$$
\dot{z}_{1}=-\rho_{z_{1}}^{-1} \rho_{z_{2}} \varphi_{2}-\ldots-\rho_{z_{1}}^{-1} \rho_{z_{n}} \varphi_{n}+\sum_{k} P_{1 k} \varphi_{k}+\sum_{k} P_{2 k} \bar{\varphi}_{k}
$$

where $P_{1 k}$ and $P_{2 k}$ are integral operators. Expressing $\dot{z}\left(\zeta_{0}\right)$ in terms of $\varphi$ by (8), (17), we get from $(16)$

$$
\begin{aligned}
& -\rho_{z_{1}}^{-1} \rho_{z_{2}} \varphi_{2}\left(\zeta_{0}\right)-\ldots-\rho_{z_{1}}^{-1} \rho_{z_{n}} \varphi_{n}\left(\zeta_{0}\right)=\sum d_{j}^{1} \varphi_{j}\left(\zeta_{0}\right)+\sum_{j} d_{j}^{2} \overline{\varphi_{j}\left(\zeta_{0}\right)} \\
& +\sum_{k} \iint_{\mathbb{D}} b_{1 k}(\zeta) \varphi_{k}(\zeta) d \zeta \wedge d \bar{\zeta}+\sum_{k} \iint_{\mathbb{D}} b_{2 k}(\zeta) \overline{\varphi(\zeta)} d \zeta \wedge d \bar{\zeta}
\end{aligned}
$$

for some $b_{1 k}, b_{2 k} \in L^{1}(\mathbb{D})$. As in lemma 6.2 we obtain

$$
\begin{gathered}
d_{j}^{1}=-\left.\rho_{z_{1}}^{-1} \rho_{z_{j}}\right|_{\zeta=\zeta_{0}}, j=2, \ldots, n \\
d_{j}^{2}=0, j=2, \ldots, n
\end{gathered}
$$

Since $\zeta_{0} \in b \mathrm{D}$ is arbitrary, we have

$$
\sum_{j=1}^{n} \rho_{z_{j}} \dot{z}_{j}=0
$$

on $b \mathrm{D}$. This precisely means that $\dot{z}(\zeta) \in H_{z(\zeta)}^{J} E$ (the holomorphic tangent space) for any $|\zeta|=1$.

By the hypothesis of proposition this is true for every disc close to $z_{0}$. By the Rank Theorem, the image of the evaluation map $\mathcal{F}_{\zeta_{0}}$ is a $J$-complex hypersurface contained in $E$. This completes the proof of the proposition. 


\subsection{Proof of the main result}

It is convenient to begin with the proof of a weaker version of our main result.

Proposition 6.3 Let $E$ be a real hypersurface in an almost complex manifold $(M, J)$. Assume that the set of points where the Levi form of $E$ vanishes identically has the empty interior and $E$ contains no J-complex hypersurfaces. Then Bishop discs of E fill a one-sided neighborhood of every point of $E$.

Proof : If $E$ admits a transversal Bishop disc attached at $p$, then the statement follows by Proposition 3.2. Suppose that there are no transversal Bishop discs. Then by Theorem 3.5 the Levi form of $E$ (with respect to $J$ ) vanishes on the boundary of every Bishop disc attached to $E$ at $p$. If these discs fill an open subset $\Omega$ of $E$, the Levi form of $E$ vanishes on $\Omega$ identically which contradicts to the assumption of theorem. Finally, if the boundaries of Bishop discs do not cover an open piece of $E$, then Proposition 6.1 implies the existence of $J$-complex hypersurfaces in $E$. Thus, $E$ necessarily admits a transversal Bishop disc which proves Proposition 6.3.

Proof of Theorem 1.1: The proof goes along the lines of that of Proposition 6.3. We only describe the necessary adjustments. We assume that there is no transverse Bishop disc attached to $E$ at $p$ and bring this to a contradiction.

To obtain a complex hypersurface passing through $p=e_{n}$ in Proposition 6.1 we use a disc $z_{0}$ with $z_{0}\left(\zeta_{0}\right)=z_{0}(1), \zeta_{0}=-1$. To obtain such a disc we take an arbitrary embedded Bishop disc $f$ and put $z_{0}(\zeta)=f\left(\zeta^{2}\right)$.

Therefore in proving most auxiliary results, we have to deal with Bishop discs $z$ close to the disc $z_{0}: \zeta \mapsto \zeta^{2} e_{n}$. Such discs generally cannot be straightened by a diffeomorphism in the proof of Lemma 2.2 on the normalization of the matrix $A$ along a disc, so we need a suitable version of the lemma. In the new version we require that the conditions $A=0$, $A_{z}=0$ be satisfied on $z\left(\overline{\mathbb{D}}_{0}\right)$, where $\mathbb{D}_{0} \subset \mathbb{D}$ is a fixed subdomain so that $b \mathbb{D}_{0}$ contains an open arc $\gamma \subset b \mathrm{D},-1 \in \gamma$, and the map $\zeta \mapsto \zeta^{2}$ is a diffeomorphism on $\mathbb{D}_{0}$. We also require that $A\left(e_{n}\right)=0$. The needed version easily follows from existing Lemma 2.2.

With the above normalization of $A$, the proof of all the results will go through with obvious adjustments. Some relations will hold only on $\mathbb{D}_{0}$ or $\gamma$. For instance, the formulas (3) and (4) will hold only on $\mathrm{D}_{0}$. In the equivalence relation in Definition 4.2 we will change the target space to $C_{1}^{2+\alpha}(\gamma)$. In the proof of Proposition 3.5 we obtain that the Levi form of $E$ vanishes on $z_{0}(\gamma)$.

Theorem 1.1 is proved.

\section{References}

[1] M. Audin, J. Lafontaine, Ed., Holomorphic curves in Symplectic geometry, Birkhauser, 1994. 
[2] M.S.Baouendi, L.P.Rothschild, J.-M.Trépreau, On the geometry of analytic discs attached to real manifolds, J. Diff. Geom. 39(1994), 379-405.

[3] E. Bishop, Differentiable manifolds in complex Euclidean space, Duke Math. J. 32 (1965), 1-21.

[4] E. M. Chirka, An introduction to the theory of $C R$ manifolds, Russian Math. Surveys, 46 (1991), 95-197.

[5] M. Gromov, Pseudo-holomorphic curves in symplectic manifolds, Invent. Math. 82(1985), 307-347.

[6] S. Ivashkovich, J.-P. Rosay, Schwarz-type lemmas for solutions of $\bar{\partial}$-inequalities and complete hyperbolicity of almost complex structures, Ann. Inst. Fourier 54(2004), 23872435.

[7] N. Kruzhilin, A. Sukhov, Pseudoholomorphic discs attached to CR-submanifolds of almost complex spaces, Bull. Sci. Math. 129(2005), 398-414.

[8] D. McDuff, D. Salamon, J-holomorphic curves and symplectic topology, AMS Colloquium Publications, 52, Providence, RI, 2004, 669 pp.

[9] J. Merker, E. Porten, Holomorphic extension of CR functions, envelopes of holomorphy and removable singularities, Intern. Math. Res. Surveys, V. (2006), Article ID 28925, 287 pp.

[10] A. Sukhov, A. Tumanov, Filling hypersurfaces by discs in almost complex manifolds of dimension 2, ArXiv math. CV/0505459, to appear in Indiana Univ. Math. J.

[11] J.-M. Trépreau, Sur le prolongement holomorphe des fonctions CR définies sur une hypersurface réelle de classe $C^{2}$ dans $\mathbb{C}^{n}$, Invent. Math. 83(1986), 583-592.

[12] J.-M. Trépreau, Holomorphic extension of CR functions: a survey, in Progr. Nonlinear Diff. Equations Appl. 21, Birkhauser Boston, MA (1996), 333-355.

[13] A. Tumanov, Extending CR functions on a manifold of finite type over a wedge, Mat. Sbornik 136 (1988), 129-140.

[14] A. Tumanov, Analytic discs and the extendability of $C R$ functions, in Lecture Notes in Math. 1684(1998), 123-141.

[15] I. N. Vekua, Generalized analytic functions, Fizmatgiz, Moscow (1959); English translation - Pergamon Press, London, and Addison-Welsey, Reading, Massachuset (1962).

[16] K. Yano, Sh. Ishihara, Tangent and cotangent bundles, Marsel Dekker NY 1973. 